\title{
EDUCACIÓN AMBIENTAL EN LA ESCUELA CHILENA: EXPERIENCIAS PEDAGÓGICAS EN LA COMUNA DE SANTA BÁRBARA
}

\section{ENVIRONMENTAL EDUCATION IN THE CHILEAN SCHOOL: PEDACOGICAL EXPERIENCES IN THE COMMUNE OF SANTA BARBARA}

\section{RENATA SÁNCHEZ VALENZUELA* \\ CARLOS OSSA CORNEJO**}

Rec.: 20-09-2020. Acept.: 07-10-2020. Publ.: 18-12-2020

DOI: http://doi.org/10.29035/ucmaule.59.103

\section{RESUMEN}

Este artículo presenta una caracterización de la percepción sobre educación ambiental; en las escuelas de la comuna de Santa Bárbara, región del Biobío, que poseen un Proyecto Educativo Institucional (PEI) con sello ambiental. Esta investigación se realizó a través de la etnometodología, para identificar y describir las prácticas pedagógicas orientadas a la educación ambiental, y describir el enfoque pedagógico frente a los problemas medioambientales que poseen docentes de las escuelas participantes. Participaron nueve docentes, de cuatro establecimientos de educación rural y urbana. Dentro de los principales hallazgos, se puede apreciar que la implementación de la educación ambiental se realiza bajo una lógica instrumental, donde se abordan escasamente procesos críticos y reflexivos que originan problemas medioambientales, propuestos desde los lineamientos de la educación con miras hacia la sustentabilidad; por otra parte, destaca la presencia de características docentes que podrían asegurar su efectividad, tales como sensibilidad frente a la crisis ecológica y conciencia crítica frente a los problemas medioambientales. Se concluye que faltan herramientas pedagógicas que puedan fortalecer el éxito de su implementación, vincularse con redes que apoyen la implementación de las propuestas, y clarificar el enfoque pedagógico de los y las docentes frente los problemas medioambientales.

Palabras clave: Educación ambiental, Enfoque Pedagógico, Sustentabilidad.

* Psicóloga, Universidad del Bío-Bío. https://orcid.org/0000-0002-1234-2257 rsvpsicologa@gmail.com

** Dr. en Psicología, Universidad del Bío-Bío. https://orcid.org/0000-0002-2716-2558 cossa@ubiobio.cl 


\section{ABSTRACT}

This article presents a characterization of the perception of environmental education, in the schools of the commune of Santa Bárbara, Biobío region, which have an Institutional Educational Project (P.E.I) with an environmental seal. This research was carried out through ethnomethodology, to identify and describe pedagogical practices aimed at environmental education, and describe the pedagogical approach to environmental problems that teachers from participating schools have. Nine teachers from four rural and urban education establishments participated. Among the main findings, it can be seen that the implementation of environmental education is carried out under an instrumental logic, where critical and reflective processes that cause environmental problems are scarcely addressed, proposed from the guidelines of education with a view to sustainability; On the other hand, it highlights the presence of teaching characteristics that could ensure its effectiveness, such as sensitivity to the ecological crisis and critical awareness of environmental problems. It is concluded that pedagogical tools are lacking that can strengthen the success of their implementation, link with networks that support the implementation of the proposals, and clarify the pedagogical approach of teachers to environmental problems.

Key words: Environmental education, Pedagogical Approach, Sustainability.

\section{INTRODUCCIÓN}

La crisis ecológica mundial es una problemática que afecta a todas las personas, independiente de su lugar de origen. Desde hace aproximadamente cuarenta años, se han gestado instancias internacionales y nacionales, con la finalidad de sentar las bases para afrontar esta realidad. Es por esto que un camino indispensable, junto con la implementación de políticas públicas y de desarrollo, es instaurar acciones desde la educación formal, considerándolo como el camino idóneo por excelencia. La escuela, con su rol formador de ciudadanos y ciudadanas reflexivos, conscientes y sensibles a las problemáticas de la sociedad, presenta una oportunidad única para abordar de manera trasversal la educación ambiental.

La educación ambiental se define como una corriente de pensamiento y acción guiada por variados enfoques teóricos y metodológicos, los que pretenden la comprensión de las realidades ambientales y el proceso histórico que ha 
conducido a la actual crisis ecológica mundial (Alea \& Jaula, 2005). Su objetivo fundamental es lograr que las personas a nivel individual y colectivo comprendan la interdependencia y complementariedad con el medioambiente, adquiriendo valores, habilidades y prácticas para prevenir y solucionar los problemas medioambientales (Organización de las Naciones Unidas para la Educación, la Ciencia y la Cultura, s.f.).

En relación a lo anterior, se puede agregar que, respecto al estado de implementación de la educación ambiental, en las escuelas de la comuna de Santa Bárbara que poseen un sello ambiental, este se caracteriza principalmente por acciones en relación a la creación de espacios ambientales escolares, tales como estaciones de reciclaje, huertos escolares y espacios promocionales e informativos en pro del medioambiente, entre otros, iniciativas que logran escasamente ser traspasadas a estrategias pedagógicas para incluir la educación ambiental dentro del aula.

Por lo anterior, es que el presente artículo pretende aportar una caracterización sobre cómo se perciben las experiencias pedagógicas en educación ambiental, dando cuenta en qué medida las escuelas de esta localidad han incorporado en sus prácticas educativas la dimensión ambiental orientada hacia la educación sustentable declarada en sus Proyectos Educativos Institucionales.

\section{MARCO TEÓRICO}

El término educación ambiental, se define como el "proceso permanente de carácter interdisciplinario, destinado a la formación de una ciudadanía que reconozca valores, aclare conceptos y desarrolle las habilidades y las actitudes necesarias para una convivencia armónica entre seres humanos, su cultura y su medio biofísico circundante" (Ley 19.300 Bases Generales del Medio Ambiente, 1994, p.2)

Por otro lado, el concepto sustentable es un derivado del constructo de desarrollo sustentable, que se entiende como el proceso por el cual se pretende preservar los recursos naturales para las futuras generaciones. Sin embargo, no considera las necesidades sociales y/o culturales que esto conlleva (Iniciativa Carta de la Tierra, 2018). 
A modo de síntesis al referimos a educación ambiental con miras hacia la sustentabilidad, aludimos a que esta tiene como objetivo el desarrollo sustentable de la sociedad, educando a las futuras generaciones partiendo de la premisa de una relación interdependiente entre las personas y la naturaleza.

\section{Educación a mbiental en el mundo}

A partir de las discusiones mundiales sobre los problemas medioambientales, es que los planes educativos de diversos países ven como tarea indispensable comenzar a incorporar dentro de sus labores, elementos curriculares y de gestión respecto a la educación ambiental.

Dentro de sus objetivos fundamentales se encuentra superar el antropocentrismo de las pedagogías tradicionales, privilegiando un enfoque y visión ecopedagógica, "basada en el entendimiento planetario de género, especies, reinos, (...) que evoluciona de una visión antropocéntrica a un entendimiento planetario, a ejercer una ciudadanía planetaria y a una nueva referencia ética y social: la civilización planetaria" (Antunes \& Gadotti, 2006, p. 142), lo que claramente requiere de un cambio radical en los procesos formativos.

Uno de los países que ha logrado una excelente incorporación del enfoque ambiental con miras hacia la sustentabilidad dentro de sus procesos educativos es Suecia, reconocido a nivel mundial como cuna de la educación ambiental debido a ser sede, en el año 1972, de la conferencia de Naciones Unidas sobre el Medio Ambiente, encuentro que marcó el punto de partida de la política internacional en relación al medioambiente. Junto con este hito, Suecia se posiciona como el país más sustentable del mundo, con una serie de políticas de sustentabilidad en las diversas áreas de desarrollo, incluyendo al currículum educativo (Ministerio del Medio Ambiente (MMA), 2018), dónde el contenido se aborda de manera eficiente y trasversal en todas las disciplinas pedagógicas.

Por otra parte, está Canadá, país que se ha convertido en el anfitrión de las cátedras de la Organización de las Naciones Unidas para la Educación, la Ciencia y la Cultura, en cuanto a la integración exitosa de la educación ambiental en la educación superior. Además, cuenta con una red nacional que reúne a sus provincias en relación a la Educación del Desarrollo Sostenible, compuesta por grupos de trabajo y educadores (Montero, 2012). 
Finalmente, en el caso de Latinoamérica y el Caribe, se encuentra Costa Rica, donde entre los años 1987 y 1988, generaron dos proyectos en pos del desarrollo sustentable, los cuales aún a la fecha siguen siendo relevantes, considerados pioneros en establecer este tipo de políticas. El primero, conocido como "La Estrategia Nacional para la Conservación y Desarrollo Sostenible" (ECODES) y el segundo, "El Plan Maestro de Educación Ambiental", iniciativas que guían una serie de acciones ambientalistas para ser incorporadas dentro de la educación (Serrano, Morales \& Chaves, 2016), incluyendo la dimensión ambiental en los distintos niveles de la enseñanza.

\section{Educación ambiental en Chile}

En octubre del año 1968 surge en Chile la primera organización ambiental, denominada "Comité de Pro Defensa de la Fauna y Flora", la cual dedicó su trabajo en actividades investigativas y educacionales, con el objetivo sensibilizar a la ciudadanía frente a la contaminación y la crisis ambiental. Luego, en la década de los 80estas organizaciones proliferaron, constituyéndose la Red Nacional de Acción Ecológica (MMA, 2018), agrupando a un sinfín de organizaciones en pro del cuidado medioambiental que establecieron las bases de la educación ambiental en Chile.

Posteriormente, en el año 1990 se promulga el Decreto 240 y se crea la Comisión Nacional Del Medio Ambiente. En 1994 se crea la ley 19.300, donde el Estado asume como deber la promoción de la educación ambiental. En el mismo año, se crea el departamento de educación y capacitación ambiental, lo que marcaría un hito en el país, debido a que vinculó la educación ambiental y la educación ciudadana (MMA, 2018), uniendo en esta iniciativa a la Corporación Nacional Forestal y al Ministerio de Educación.

En relación al ámbito curricular, en el año 1996 el Ministerio de Educación, dentro de la reforma curricular, incorpora en el currículum nacional la dimensión de la educación ambiental de manera transversal en todas las áreas de conocimiento, específicamente en los Objetivos Transversales de la Educación (OFT). Estos se encuentran agrupados en 4 dimensiones, donde la Dimensión de convivencia escolar, que apunta a formar personas como ciudadanos democráticos comprometidos con su entorno, evidencia la importancia de educar a los estudiantes en relación a los problemas medioambientales. La 
relevancia de esto "radica en el propósito de alcanzar un equilibrio entre una educación instrumental que prepare a los niños, niñas y jóvenes para su inserción laboral, académica y una educación que contribuya a formar personas responsables consigo mismos y los otros" (Departamento de Educación Ambiental y Participación Ciudadana (CONAMA), 2006, p.10), lo cual refleja la iniciativa de incluir la temática dentro del campo educativo, con proyecciones hacia una educación para la sustentabilidad.

En el año 2004 y siguiendo el objetivo de incorporar en los planes educacionales vigentes la dimensión medioambiental, se crea el Sistema Nacional de Certificación Ambiental de Establecimientos Educacionales, que pretende generar aprendizajes situados, que respondan a una visión planetaria de los fenómenos naturales y sociales y que promueva la construcción de proyectos educativos integrales (Departamento de Educación Ambiental y Participación Ciudadana-CONAMA, 2006). El proceso de Certificación Ambiental de Establecimientos Educacionales, tiene como objetivo fundamental

aportar un modelo para las escuelas que implica determinar los ámbitos centrales de la acción pedagógica y escolar en que es posible incorporar contenidos y prácticas de la educación ambiental, que contribuyan al mejoramiento de la formación de los niños, niñas y jóvenes (Departamento de Educación Ambiental y Participación CiudadanaCONAMA, 2006, p.18).

Por último, en materia internacional, se destaca que en 2015 Chile se une, junto a otros 193 países, a la agenda 2030, la cual establece los objetivos para el desarrollo sostenible, iniciativa que responde a un plan de acciones asociado a metas desde la educación formal, que apuntan a la calidad, producción, consumo y responsabilidad de los ecosistemas terrestres (MMA, 2018). Finalmente, cabe destacar que el 2017 de generó un diagnóstico, por parte del Ministerio de Desarrollo Social de Chile, en relación a los objetivos de la agenda 2030, teniendo como resultado un estado de avance significativo en la mayoría de las metas propuestas (MMA, 2018).

\section{Participa ción docente en la implementación de la educación ambiental}

La educación ambiental se basa mayormente en políticas establecidas, más que en tendencias impulsadas por la innovación. La reinterpretación que realizan los 
países adheridos a los tratados internacionales, en relación al concepto de educación ambiental, es la que guía sus políticas educativas (Nguyen, 2019).

Lo anterior, genera diferentes perspectivas e interpretaciones al respecto, conllevando a diferentes formas de implementar los acuerdos internacionales en el contexto educativo local. Sin embargo, existe un punto en común dentro de esta mirada multifacética, el cual radica en la importancia del rol del docente en la implementación de los principios de la educación ambiental con miras a la sustentabilidad, debido a que ellos son quienes transmiten directamente el discurso de la sustentabilidad desde la política nacional hacia la política en práctica (Nguyen, 2019).

La educación ambiental es reconocida por su calidad polisémica, construida en base a una serie de conocimientos, valores y prácticas que la complejizan, lo que se traduce en variados elementos que caracterizan su plan de estudios, los cuales abarcan el uso de conceptos científicos y conceptos sociales, tales como consenso social, prácticas sociales, etc. (Ndiaye, Khushik, Diemer \& Pellaud, 2019). Esto complejiza aún más su incorporación en la educación formal y en el currículum, refiriendo los y las docentes que la educación sustentable es difícil de conceptualizar, debido a ser muy abstracta (Ates \& Gül, 2018), lo que implementarla se convierte en un desafío profesional.

Dada esta complejidad, así como la existencia de diversos enfoques pedagógicos frente a los problemas medioambientales, es que algunos autores plantean la relevancia de incorporar la formación en educación ambiental con miras a la sustentabilidad desde la formación inicial docente. De esta forma, los futuros docentes podrán acceder a la información, conocer los paradigmas a la base y contar con las herramientas teórico-metodológicas para poder impartir la educación ambiental a sus futuros estudiantes (Torres-Porras \& Arrebola, 2018), basándose en una lógica que vaya en congruencia con las políticas públicas creadas para abordar el tema, dentro del contexto nacional.

La formación inicial docente, en cuanto a la incorporación de la educación ambiental con miras a la sustentabilidad, tiene una tarea pendiente. Esta carece de manuales metodológicos sobre las formas de implementación en la educación escolar (Wojciech, 2018), lo que sin duda lo vuelve un elemento crucial de ser abordado en la formación universitaria. 


\section{Enfoques pedagógicos frente a los problemas medioambientales}

Existen tres enfoques pedagógicos frente a los problemas medioambientales (Ávila, Molina \& Pagola, 2018). El primero, corresponde a un enfoque ecológico, basado en una tradición educativa técnica, regida por un paradigma antropocéntrico, dónde los problemas medioambientales son vistos como de índole productivo-económico. Las actividades de aprendizaje son de tipo hipotético deductivas, utilizando generalmente definiciones y conceptos con escaso o nulo componente reflexivo. En segundo lugar, se encuentra el enfoque pedagógico sustentable frente a los problemas medioambientales, responde a una tradición constructivista, donde los problemas medioambientales se perciben como limitados, incorporando además una mirada global de dicha problemática. Este enfoque se encontraría en un proceso intermedio entre un enfoque ecológico y un enfoque para la sustentabilidad y posee una visión social progresiva donde se potencia la educación ambiental del ciudadano, pero no se acompaña de medidas más profundas de transformación social (Ávila et al., 2018), es decir, se analizan los problemas medioambientales, pero no se llevan a cabo acciones concretas para su afrontamiento.

Finalmente, el enfoque para la sustentabilidad, se basa en la tradición sociocrítica de la educación, enmarcado dentro de un paradigma biocéntrico (García, 1999), el cual establece que la relación entre el medioambiente y la humanidad se genera en base a una interdependencia y complementariedad, donde la didáctica propuesta supone el uso de estrategias de aprendizaje basadas en procesos analíticos de autoconciencia y comunicación, que propicien el debate entre los argumentos propios y ajenos, posibilitando la reflexión y favoreciendo el desarrollo de una actitud crítica transformadora (García, 2004), es decir, se transita desde la reflexión crítica hacia la práctica en acción.

\section{METODOLOGÍA}

La investigación se centró en un paradigma comprensivo/interpretativo, utilizando un método cualitativo. Como metodología se utilizó la etnometodología, la más idónea para dar respuesta a la pregunta de investigación desde el sentido y las acciones cotidianas que realizan las personas en un contexto determinado, en este caso docentes de una institución educativa. 
El contexto de la investigación corresponde a un grupo de 4 escuelas municipales correspondientes a la comuna de Santa Bárbara, provincia del Biobío, Chile. En primera instancia se solicitó autorización al jefe del Departamento Municipal de Educación, para luego invitar a todos los establecimientos de la comuna a formar parte del trabajo investigativo, para finalmente llegar a un número en total de 4 establecimientos que manifestaron de forma voluntaria su interés en participar.

Los y las participantes fueron 9 docentes de diversas asignaturas que se imparten dentro de las escuelas participantes, los y las cuales forman parte del establecimiento con una data mínima dos años. Estos colaboraron de forma voluntaria en la investigación a través de un muestreo por acceso.

Con respecto a las técnicas de recolección de información se trabajó con entrevistas semiestructuradas, con un total de 10 preguntas abiertas centradas en indagar información sobre la temática investigada. Junto con esta, se realizó revisión documental de los instrumentos de gestión educativa, registro fotográfico de las instalaciones escolares y observación no participante. Esta información se analizó mediante el análisis de contenidos explícitos e implícitos con el fin de generar la reducción de datos, a través de la categorización y codificación, dando paso a la disposición y transformación de la información (Rodríguez, Lorenzo \& Herrera, 2005). Para esto, se realizó un trabajo de análisis de contenido temático para la generación de categorías emergentes. Finalmente, luego de la saturación de contenido se confeccionó la presentación de resultados y la posterior triangulación de la información y conclusiones.

\section{RESULTADOS}

A partir del análisis de contenido, se obtuvieron 5 categorías de estudio.

\section{Orientaciones a mbientales en los PEI}

El Proyecto Educativo Institucional (PEI) es el marco teórico donde se asientan los objetivos pedagógicos propios de la escuela. En este se plasma su visión, misión, sello y se definen sus principios y valores morales y académicos. Concretamente el PEl es la carta de presentación de una escuela, el cual refleja las particularidades de las actividades pedagógicas que guiarán sus principios educativos. 
Las intenciones de los PEl reflejan la pretensión de formar estudiantes sensibles frente a las problemáticas medioambientales y promotores del cuidado del medioambiente. En apartados de sus misiones, visiones y perfiles de estudiantes se puede encontrar la presencia de la educación ambiental como guía para alcanzar algunos sus objetivos pedagógicos.

"La Escuela aspira a posicionarse en la comuna como una institución de Educación General Básica reconocida por su calidad educativa y su alto compromiso en la formación integral de los alumnos y alumnas basada en principios humanistas y cristianos que los convierte en personas tolerantes, respetuosas de la diversidad, protectores y promotores del cuidado del medio ambiente, solidarios, reflexivos, críticos, autónomos y con espíritu emprendedor" (P.E.I. ESCUELA C).

"Sostenemos que es responsabilidad de todos los miembros de la comunidad educativa, el cuidado del medio ambiente natural y además ser propulsor de actitudes positivas en el entorno social en que se desenvuelven, ya sea: su familia, sus amigos, su barrio, su curso, su escuela, etc." (P.E.I. ESCUELA J).

"Entregar un servicio educativo de vanguardia pedagógica a todos los estudiantes valorando su contexto, y comprometidos con los principios de la educación Ambiental" (P.E.I ESCUELA H).

"Con un fuerte énfasis en el emprendimiento e innovación utilizando como base fundamental el desarrollo del lenguaje y la comunicación en toda su extensión, la música y la actividad física como complemento y la actividad hortofrutícola como herramienta para la vida", donde agregan en el perfil de sus estudiantes la pretensión de que estos y estas sean "Responsables y comprometidos en todo su quehacer diario y su entorno" (P.E.I. ESCUELA N).

\section{Implementación de las activida des de aprendizaje a mbiental}

Esta categoría se define como las acciones ambientales llevadas a cabo en los establecimientos educativos. Se puede señalar que las orientaciones propuestas en los PEI se reflejan en las actividades de aprendizaje impartidas por el cuerpo docente, las que dan cuenta de los lineamientos que definen tareas escolares 
realizadas fuera y dentro del aula, que hacen alusión a la educación ambiental, así como los recursos pedagógicos utilizados para llevar a cabo las actividades.

En relación a lo anterior, se observa que los contenidos medioambientales se implementan preferentemente de forma teórica a través del uso del texto escolar, en actividades que consideran el reconocimiento de conductas sociales o prácticas afines al cuidado medioambiental.

"Me refiero por ejemplo que, si usted lee un texto que dice que los zorros se están acabando en el cerro el xx, pero aparece aquí no más, ¿y lo leyó nada más que hizo con eso el alumno? Nada..." (Prof. D. M., Escuela H).

"Está presente el tema medioambiental, pero de forma utilitaria, o sea... como decirlo, lo podemos usar, pero como un tema para explicar otros contenidos" (Prof. R. R., Escuela C).

Junto con lo anterior, se observa que el material de apoyo pedagógico se corresponde con lecturas basadas en ejemplos que presentan contenido del tema abordado de manera instrumental, medianamente insuficiente en comparación a los lineamientos propuestos desde la educación ambiental con miras a la sustentabilidad.

\section{Espacios de implementación de la educación a mbiental}

Otro aspecto que da cuenta de la implementación de los lineamientos de los PEl, se refiere a los espacios físicos que reflejan la presencia de la dimensión ambiental en las actividades escolares, las que hacen referencia a huertos escolares y espacios de promoción del cuidado del medioambiente.

Los establecimientos educacionales han implementado espacios para el desarrollo de la educación ambiental, los cuales son principalmente afiches promocionales para la creación o adecuación de la conducta en función del cuidado de la naturaleza, y la construcción de huertos escolares. Respecto a los huertos escolares, se observa que en su mayoría han quedado relegados a espacios que cumplen funciones de acopio de material o mobiliarios. Se puede agregar que la mantención de estos espacios generalmente depende del interés de la escuela por llevar a cabo actividades en relación a la dimensión medioambiental. 
Por otro lado, respecto a las estructuras y funcionamiento de huertos escolares, los y las docentes entrevistados refieren que:

"No tenemos los espacios para hacerlo. En nuestra escuela se estuvo haciendo por ejemplo huertos escolares, plantación de arbolitos, pero se queda en eso, el docente que estaba a cargo se fue..." (Prof. L. M., Escuela C).

"Tenemos los espacios para el huerto, es más yo tengo horas para eso, pero se me pidió que las utilizara en talleres destinados a otros fines" (Prof. R. R., Escuela C).

Junto a lo anterior, si el interés de la escuela es positivo, en relación a la incorporación de la dimensión medioambiental en sus procesos educativos, la responsabilidad de la implementación de los espacios físicos, su cuidado y mantención recae generalmente en un número reducido de docentes con horas asignadas para aquello, de preferencia docente de ciencias, no en la comunidad educativa en general, por lo que, si el docente encargado de realizar esa labor cesa sus funciones en la escuela, los espacios terminan abandonados.

"Esa bueno... he... hay muchos profesores que no le gusta esto de la tierra, del huerto de las plantas... todos debemos estar participando de esta acción, acá como la escuela tenía certificación medioambiental, por todas esas cosas que habíamos hecho antes con otras colegas que se fueron, y se fueron por esa misma mala conducta de la directiva... por ejemplo, la señora xx, éramos tres los encargados, ella manejaba los compost... era profesora, hacía ramos técnicos..." (Prof. D. M., Escuela H). "Si, retomando... lo que hubo años atrás... y también se requiere que los profesores se comprometan en general a hacer cosas, y debido a eso mismo acá en la escuela se fueron todos los profesores que eran antiguos... se empezó a ir la gente..." (Prof. D. M., Escuela H).

\section{Prácticas pedagógicas en educación ambiental}

Esta categoría corresponde a la descripción más detalladas de las prácticas pedagógicas presentes en la escuela desde la visión del docente, referidas a su rol y su opinión sobre instancias de formación pedagógica en la temática medioambiental, su percepción en cuanto a las habilidades y conductas por parte 
de los educandos, además de una evaluación temporal de la implementación de la educación ambiental en el currículum oficial.

A partir de los datos generados, se relevan las siguientes subcategorías:

\subsection{Instancias de capacitación docente en educación a mbiental}

Esta categoría se define como las instancias de capacitación en educación ambiental para los y las docentes, correspondiente a actividades de capacitación profesional, tanto durante su formación inicial o en su ejercicio profesional.

Según refieren los y las entrevistadas, estas han decaído producto de la implementación de la Jornada Escolar Completa (JEC), hecho relevante, debido a que el objetivo de la JEC, precisamente, busca la formación de los y las estudiantes desde una perspectiva integral.

"Cuando se instaló la JEC, jornada completa, había que hacer talleres, porque las horas de la tarde son talleres, entonces esas dos horitas de taller que cada profesor tenía la ocupábamos en el huerto y se llevan a los niños se fueron acabando, se hacían esas cosas en ese tiempo, entonces la escuela fue bien nombrada en ese aspecto porque nosotros fuimos bien activos en esta parte de enseñarle a los niños todo esto que fuera sano..." (Prof. D. M., Escuela H).

Las nuevas orientaciones en el plan educativo se aplicaron correctamente, pero al transcurrir el tiempo nuestro país asentó esta innovación bajo la lógica de los resultados, las habilidades y las pruebas estandarizadas, lo que conllevó al decaimiento de prácticas enfocadas anteriormente a talleres medioambientales. Por ende, capacitarse en temáticas medioambientales queda relegado a una necesidad que depende del interés del o la docente y de las oportunidades que brinde el establecimiento, hecho que ha evolucionado negativamente de acuerdo a lo relatado.

"Decayó porque hubo como un cambio por que le dieron más importancia al lenguaje a las matemáticas y ya los talleres no pasaron a ser talleres de arte o huerto, de música, sino que todo estaba enfocado a taller enfocado en lenguaje matemática..." (Prof. D. M., Escuela H).

"Creo que hubo un descenso, como que se dejó de lado, no solo lo referente al medio ambiente y las actividades propias de la vida rural, 
sino que, en las artes y otras cosas, ahora el foco está en los resultados en rendimiento..." (Prof. R. R., Escuela C).

Por otro lado, en relación a la formación inicial docente de los y las entrevistados, declaran haber recibido escasamente la formación pedagógica y didáctica que permita implementar la dimensión medioambiental.

“Mm... no... que podríamos relacionarlo a eso... una visita a un invernadero, que podríamos relacionarlo más con esto, pero fue una visita más de ciencias... teníamos un ramo de ciencias que ni recuerdo cómo se llamaba, pero fuimos a un invernadero..." (Prof. B. .M, Escuela J).

Se observa que los y las docentes con mayores años de servicio, poseían antaño mayor libertad de acceder a actividades de capacitación enfocadas a la educación ambiental, junto con contar con facilidades para destinar tiempo para su implementación en las designadas horas de "taller", las que en la actualidad se enfocan principalmente a fortalecer habilidades evaluadas por Sistema Nacional de Medición de Calidad de Educación (SIMCE).

"Bueno, si... yo antes, antes nosotros íbamos a muchos cursos, yo hice cursos de huertos escolares, yo hice más de 1000 horas de huertos escolares (...) lo hice en la universidad de concepción, también estuve participando en el instituto IEP de Santa Fe, instituto de educación rural, ahí hicimos el curso de fruticultura y capacitación de las abejas.... Y estuvimos más o menos dos años en eso..." (Prof. D. M., Escuela H).

Por último, dentro de las actividades de aprendizaje enfocadas a la educación ambiental, se reconoce el liderazgo de la disciplina de ciencias la cual releva a los y las docentes de estas áreas en la implementación de este material y de las actividades de aprendizaje medioambiental, posicionándolos como protagonistas. Se observa que él o la docente de ciencias del establecimiento, exceptuando a los y las docentes, poseen el liderazgo y responsabilidad de llevar a cabo las actividades en relación a la materia.

"En realidad todas las actividades como fuertes en el tema las vemos con el comité de ciencias, que está compuesto por la docente de ciencias, tecnología y taller" (Prof. RR, Escuela C). 


\subsection{Visión del docente sobre integración de la cultura medioambiental en el estudiantado}

A pesar del trabajo propuesto por el Ministerio de Educación y el Ministerio del Medio Ambiente, las iniciativas curriculares, junto con las iniciativas institucionales escolares de incorporar la educación ambiental en sus estudiantes, los y las docentes refieren que la mayoría del estudiantado no poseen habilidades de acción y conciencia crítica en cuanto a la problemática.

"Creo que les falta, estamos incentivando, integrando, comunicando, enseñando que esto grave, estamos como en un primer piso, ellos lo entienden, participan, pero no sé si para la acción..." (Prof. B. M., Escuela J).

Por otro lado, como información emergente, se observa que los y las docentes evidencian un comportamiento y habilidades diferentes en los niños y niñas que están egresando actualmente de educación parvularia. Aluden que estos y estas tendrían mayores capacidades de acción y conciencia frente a los problemas medioambientales.

"Se nota, de verdad que se nota, hay niños que estuvieron en el jardín de al lado y hay niños que llegaron sin jardín y entre ellos se ve la diferencia en el tema medioambiental, porque en las casas no se trabaja pu..." (Prof. B. M., Escuela J).

"Yo creo que se ve la diferencia en los pequeñitos sobre todo los que vienen del jardín xx, ese jardín tiene sello sustentable y ha sido premiado por el Ministerio" (Prof. N. F., Escuela C). 


\subsection{Reflexiones docentes sobre la educa ción ambiental}

Por reflexiones docentes se hace alusión a observaciones profundas que realizan estos y estas en torno a la implementación de la educación ambiental dentro de sus escuelas. Los y las docentes entrevistados concuerdan que la escuela debería ser protagonista en la implementación de la educación ambiental y donde toda la comunidad educativa debiese ser parte del proceso.

"Yo creo que debería ser promotora, promotora del cuidado del medio ambiente (...) educarlos, en base al reciclaje, educarlos en que tenemos que crear conciencia en ellos, porque ellos van a ser el futuro de la sociedad, entonces hay que crear buenas semillas con ellos..." (Prof. BM, Escuela J).

Además, los y las docentes coinciden que la escuela debe ser responsable en gran medida de instaurar la conciencia medioambiental en los niños y niñas, dónde el docente y toda la comunidad educativa deben ser guías en este proceso a través de actividades escolares y de aprendizajes y por sobre todo dando el ejemplo a los y las estudiantes.

Junto a lo anterior, los participantes refieren que existe una relación entre las actitudes a favor del medioambiente que emanan desde el docente y la eficacia de impartir la educación ambiental a los y las estudiantes. Es decir, si un docente despliega en su diario vivir actitudes a favor del cuidado del medioambiente estaría enseñando con el ejemplo, lo que ellos refieren ser la base para que los estudiantes puedan aprender sobre el tema. Lo anterior, lo denominan como una actitud de vida, otorgándole un estilo de vida cercano con el medioambiente, lo que se percibe como un valor agregado, por sobre otros docentes, a la hora de impartir la educación ambiental.

La incorporación de las prácticas escolares a favor del medioambiente se basa en fortalecer conductas destinadas a que los y las estudiantes logren ser promotores del cuidado del medioambiente, por lo que el interés o desinterés del docente en el tema, marcaría una diferencia crucial para lograr con éxito este objetivo propuesto en los PEl. 


\subsection{Valoración docente de las prácticas y políticas educa tivas a mbientales}

Este aparato da cuenta de cómo se evalúa por parte del docente, las acciones pedagógicas medioambientales dentro del sistema escolar.

Los y las docentes comentan que antiguamente la temática medioambiental no era recurrente en las prácticas escolares, refiriendo que la crisis ecológica no era tan evidente, o tan grave, como lo es hoy en día. Concuerdan además que existe un avance temporal positivo de la incorporación de la educación ambiental en la escuela, donde los intentos por incluirla en el currículum se han convertido en un esfuerzo constante.

"Antes no salía ni en las materias, yo soy docente de ciencias y sólo lo veíamos en una pequeña unidad, ahora no.... ahora es casi transversal que se trabaja con la temática medioambiental..." (Prof. R. R., Escuela C).

"Yo creo que los medios tecnológicos de comunicación y la contaminación que ha aumentado gravemente nos ha hecho integrar el tema en la escuela como algo urgente de ser abordado" (Prof. N. F., Escuela C).

\section{Enfoque pedagógico del docente frente a los problemas medioambientales}

Esta categoría da cuenta del enfoque pedagógico frente a los problemas medioambientales, presente en los y las docentes entrevistadas. Existen tres enfoques y cada uno responde a la conciencia ambiental que posea cada persona. El primero, refiere a un enfoque ecológico que responde a la tradición educativa técnica, que se rige por un paradigma antropocéntrico y por actividades de aprendizaje de tipo hipotético deductivo enfocado en conceptos o definiciones. El segundo refiere a un enfoque sustentable, se caracteriza por una tradición educativa constructivista, basada en un paradigma intermedio entre lo antropocéntrico y biocéntrico. Utiliza actividades de aprendizaje orientadas a la reflexión, pero no a la acción. Por último, se encuentra el enfoque para la sustentabilidad, el cual hace alusión a una tradición educativa sociocrítica, basada en un paradigma biocéntrico, conformado por actividades enfocadas en la reflexión y la acción, superando con creces el estadio de aprendizaje técnico. 


\subsection{Opción de enfoque}

En relación a las actividades de aprendizaje llevadas a cabo por los y las docentes, se observa un enfoque pedagógico de tipo ecológico frente a los problemas medioambientales en cuanto al tratamiento del contenido.

"Este año se celebró el día del árbol, el día del medioambiente; se hacen actos, dibujos, se presentan videos y también la fiesta pehuenche, también se realiza eso, porque también tiene que ver..." (Prof. L. M., Escuela C).

"Si, en las tres instancias ... organiza, por cursos... em (...) el día del árbol o agua no sé si me equivoco (...) los niños sí participaron, hicieron unas cartulinas, las pegaron afuera, se armó un cuento en relación a eso..." (Prof. B. M., Escuela J).

Estas características son reforzadas por el material de aprendizaje que aborda la temática de forma instrumental (textos escolares, correspondiente a la categoría anterior), lo que otorga un tratamiento del contenido con características teóricas, basadas en definiciones, celebraciones conmemorativas medioambientales, etc. Lo anterior corresponde a una educación más bien técnica, la cual se condice directamente con los trabajos y actividades que se Ilevan a cabo. Se observa que estas actividades apuntan escasamente al proceso reflexivo que conlleva a dar nuevo uso a los elementos reciclados, sino más bien al parecer son utilizados como materiales alternativos de manualidad para confeccionar trabajos escolares.

Por otro lado, en relación con sus prácticas en el cuidado medioambiental y su visión de estos en su vida personal, dan cuenta de acciones que responden a un enfoque sustentable y en ocasiones a un enfoque para la sustentabilidad.

"Si, en cosas básicas, yo por ejemplo tengo un recolector de aguas Iluvias en mi casa, también, a ver no sé si es reciclar, pero por ejemplo las verduras, todo lo que queda de las comidas, se las entregamos a un vecino, que animales" (Prof. P. J., Escuela N).

"Si... creo que últimamente si... (...) he tomado más conciencia y he valorado las acciones que se realizan en pos del cuidado del medio ambiente (...) tratamos de tener como estos contenedores donde echamos las botellas" (Prof. L. M., Escuela C). 
Los y las docentes manifiestan una sólida conciencia sobre los problemas medioambientales reflejadas en acciones en su vida personal, siendo para ellos y ellas una problemática urgente de ser solucionada, respondiendo con un enfoque sustentable frente a los problemas medioambientales y en ocasiones con un enfoque para la sustentabilidad, ambos implementados en su vida diaria, alejados de su rol docente.

"A nivel general yo la evalúo grave, una situación muy compleja porque nosotros las personas los seres humanos no tomamos conciencia de cuidar el medio ambiente" (Prof. L. M., Escuela C).

Sin embargo, en relación a su rol docente ocurre un cambio significativo a la hora de impartir los aprendizajes medioambientales. En este rol evidencian un enfoque ecológico, que se caracteriza por propiciar escasamente procesos reflexivos en el estudiantado.

Los y las docentes refieren que falta crear conciencia ambiental y superar el aprendizaje instrumental de los problemas medioambientales. Sin embargo, sus prácticas pedagógicas en relación a lo medioambiental, las cuales deberían ser guiadas por las propuestas de sus PEI junto con las directrices del equipo de gestión del establecimiento, no logran total coherencia para que los objetivos ahí propuestos se reflejen en las actividades de aprendizaje implementadas, y por consiguiente cumplir con el perfil de estudiantes a los cuales aspiran.

En resumen, se puede observar que los enfoques docentes frentes a las problemáticas medioambientales no responden a un solo tipo, sino más bien se ven traslapados unos con otros, en este caso de tipo ecológico en la implementación de las actividades de aprendizaje medioambiental y por otro, en su visión y tratamiento más allá del aula correspondiente a un enfoque de tipo sustentable y en ocasiones para la sustentabilidad. 


\section{CONCLUSIONES}

En relación con el análisis de los PEl de las escuelas participantes, se puede mencionar que, sus glosas concuerdan en la pretensión de formar estudiantes promotores del cuidado del medioambiente, sensibles frente a las problemáticas de su entorno. Lo anterior, conllevaría a una conciencia ecológica reflejada en acciones por parte del estudiantado, lo que se refleja escasamente en evaluación de los y las docentes en relación a la conciencia ambiental que poseen sus estudiantes, realizando un balance negativo al respecto. Esta conciencia ambiental debería ser fruto de las actividades de aprendizaje, sin embargo, estas se caracterizan por contenidos que hacen referencia a la educación ambiental a través de contenidos instrumentales, logrando escasamente los objetivos propuestos desde sus PEI, de crear y fortalecer una conciencia ambiental en los estudiantes, propiciando una actitud crítica frente a la problemática.

Es por lo anterior que, para cumplir con las orientaciones medioambientales propuestas en el PEl, es imperativo generar un trabajo en red, no sólo con respecto a la relación escuela y comunidad, sino además en concordancia entre el Ministerio de Educación, Ministerio del Medio Ambiente y la Escuela, donde se dictaminen y resguarden desde esas entidades los espacios de aprendizaje y acción en pro de lo medioambiental dentro de las actividades escolares, orientando la mirada hacia la capacitación docente, debido a la importancia crucial que tiene el rol docente en la implementación de la educación ambiental.

Con respecto a la necesidad de cumplir dichas orientaciones, cobra relevancia el proceso de capacitación docente en educación ambiental. Se puede observar que estas actividades de capacitación docente tienen un auge entre los años 1990 y 2000, donde existía una amplia oferta de capacitación docente al respecto y facilidades para acceder a ellas. Luego, en el año 1997 se instaura la Jornada Escolar Completa (JEC), hito que produjo un cambio significativo. Aunque sus propósitos apuntaban a una educación integral, entre otros, y su implementación se realizó correctamente, se observa un fenómeno de monopolio educativo, privilegiando las asignaturas tradicionales como matemáticas y lenguaje, esto producto de la aplicación de pruebas estandarizadas a las escuelas que las estratifican según su éxito en la evaluación, como es el caso de la prueba SIMCE.

Las horas de talleres destinadas que propuso la JEC, desplazaron a las antiguas actividades de talleres medioambientales o artísticos, sustituidas por actividades 
de reforzamiento que apuntaron a fortalecer habilidades propias de las áreas disciplinares. Lo anterior, es reforzado en gran medida por las decisiones de los equipos directivos de las escuelas, las cuales destinan los recursos humanos y planifican las instancias de aprendizaje, en su mayoría a las áreas que mide el SIMCE. Esto da cuenta que los lineamientos medioambientales propuestos en sus PEI, se implementan y cumplen escasamente.

Lo anterior, deja de manifiesto otra característica de la implementación de la educación ambiental, la cual sería la importancia que tiene las motivaciones de los equipos directivos en el éxito o no del cumplimiento de las tareas que se proponen desde sus PEI. Esto a su vez crea un sesgo en cuanto a las temáticas que son abordadas en las capacitaciones que se ofrecen a los y las docentes, las cuales generalmente están orientadas al manejo conductual de los estudiantes o a temas informativos sobre las nuevas actualizaciones de las leyes del Ministerio de Educación, entre otras, pero no a temáticas medioambientales y su tratamiento pedagógico.

Los y las docentes refieren que la escuela debería tener un rol protagónico en la implementación de la conciencia medioambiental en estudiantes, a través de actividades pedagógicas orientadas a fortalecer habilidades y actitudes en pos de lo medioambiental. En este rol protagónico de la escuela, que lidera principalmente el docente, el logro de estos objetivos se ven fortalecidos por una característica especial que tendría el educador con mayor éxito en este propósito, la cual sería la relación cercana con el entorno rural en su vida personal. Esto al parecer le otorgaría un estilo de vida cercano con el medioambiente, dotándolo de habilidades particulares para impartir el contenido ambiental.

Según los relatos de los y las docentes, en sus vidas como ciudadanos, estos refieren la urgencia de generar acciones que se antepongan a la crisis ecológica del país y del mundo, por lo que inevitablemente sus prácticas docentes han sido teñidas con esta necesidad, lo que tiene concordancia con una de las cualidades que debe tener el docente con orientación medioambiental, propuesto por el Ministerio del Medio Ambiente de Chile, que es: poseer una actitud coherente a los valores que propone la educación ambiental, para de esa formar poder transmitirlos al estudiantado (Muñoz-Pedreros, 2014). Sin embargo, el puente de esta conciencia ecológica personal con la actividad docente como tal, se encuentra escasamente fortalecido, dejando en evidencia la falta de 
herramientas pedagógicas y sobre todo político educativas que fortalezcan el abordaje transversal del contenido en la escuela.

Lo anterior, genera la necesidad de conocer cuál es el enfoque pedagógico del docente frente a los problemas medioambientales, debido a que según sus características marca la diferencia en el tratamiento del contenido y por consiguiente trastoca los resultados de aprendizaje.

En relación con los enfoques, se puede apreciar en la información aportada por los y las entrevistadas, un enfoque sustentable y en ocasiones para la sustentabilidad, el cual hace referencia a la instauración de una conciencia ecológica guiada por la reflexión crítica y la acción. Estas prácticas son recurrentes en los y las docentes dentro de su vida personal, pero no logran traspasarla al aula, lo que conlleva a observar un doble enfoque del docente frente a los problemas medioambientales.

Este fenómeno da cuenta de un camino entrecortado. Los y las docentes tienen nociones de un enfoque sustentable, pero a la hora de su implementación pedagógica utilizan un enfoque ecológico, posiblemente ocasionado por la escasa formación en herramientas pedagógicas para implementar la educación medioambiental. Sin embargo, es importante mencionar que, a pesar de esta debilidad, los y las docentes participantes presentan una cualidad fundamental que podrían asegurar el éxito de la implementación medioambiental, que es una actitud valórica hacia el cuidado del medioambiente. Por ende, es esencial que se logre romper con el estereotipo del docente tradicional para de esta forma convertirse en un facilitador de cambios con el fin de lograr formar ciudadanos y ciudadanas que logren aportar y transformar a la sociedad y que se le otorgue mayor relevancia a la formación en educación ambiental como eje transversal en la docencia a partir de la formación inicial y posteriormente facilidades de acceso y oferta de formación continua en esta área. 


\section{REFERENCIAS BIBLIOGRÁFICAS}

Alea, A., \& Jaula, J. (2005). La educación ambiental desde la pedagogía social en el contexto latinoamericano. Pensamiento Educativo. Revista de Investigación Educacional Latinoamericana, 37(2), 296-310. Recuperado de http://pensamientoeducativo.uc.cl/index.php/pel/article/view/369/782

Antunes, Á., \& Gadotti, M. (2006). La ecopedagogía como la pedagogía indicada para el proceso de la Carta de la Tierra. La Carta de la Tierra en Acción. Hacia un mundo sostenible. Ámsterdam: Kit Publishers, pp. 141-143. Recuperado de https://earthcharter.org/wp-content/assets/virtuallibrary2/images/uploads/Antunes.pdf

Antunes, Á., \& Gadotti, M. (2006). La ecopedagogía como la pedagogía indicada para el proceso de la Carta de la Tierra. BLAZE, P.; VILELA, M.; ROERINK, A. La Carta de la Tierra en acción. Hacia un mundo sostenible. Ámsterdam: Kit Publishers, 141-143. Recuperado de https://earthcharter.org/wp-content/assets/virtual-

library2/images/uploads/Antunes.pdf

Ates, H., \& Gül, S. K. (2018). Investigating of Pre-Service Science Teachers' Beliefs on Education for Sustainable Development and Sustainable Behaviors. International Electronic Journal of Environmental Education, 8(2), 105122. Recuperado de https://files.eric.ed.gov/fulltext/EJ1181067.pdf

Ávila, C., Molina, M., \& Pagola, L. (2018, 11 de diciembre). Ciudadanía Activa: Reflexiones sobre la necesidad de transitar hacia una Educación para la Sustentabilidad. El caso del Liceo Ríos de Chile de Lirquén desde la Sistematización de Experiencias con enfoque educativo. En Encinas, F., Arze, G. y Fuentes, P [Presidencia]. III Congreso de Investigación Interdisciplinaria en Arquitectura, Diseño, Ciudad y Territorio. Santiago de Chile.

Congreso Nacional de Chile (1994, 9 de marzo). Ley Nº 19300. Aprueba ley sobre bases generales del medioambiente. https://www.leychile.cl/Navegar?idNorma=30667 
Departamento de Educación Ambiental y Participación Ciudadana-CONAMA. (2006). Guía para la Implementación Pedagógica de la Educación Ambiental. Santiago. https://educacion.mma.gob.cl/wpcontent/uploads/2015/09/Guia_para_la_implementacion_Pedagogica_d e_la_Educacion_Ambiental.pdf

García, J. (1999). Una hipótesis de progresión sobre los modelos de desarrollo en Educación Ambiental. Revista Investigación en la Escuela, 37, 15-32. https://idus.us.es/xmlui/bitstream/handle/11441/60073/Una\%20hip\%C3\% B3tesis\%20de\%20progresi\%C3\%B3n\%20sobre\%20los\%20modelos\%20d e\%20desarrollo\%20en\%20Educaci\%C3\%B3n\%20Ambiental.pdf?sequenc $\mathrm{e}=1$

García, J. (2004). Los contenidos de la Educación Ambiental: una reflexión desde la perspectiva de la complejidad. Revista Investigación en la Escuela, 53, 31-51.

https://idus.us.es/xmlui/bitstream/handle/11441/61001/Los\%20contenidos \%20de\%20la\%20Educaci\%C3\%B3n\%20Ambiental.\%20una\%20reflexi\%C3 \%B3n\%20desde\%20la\%20perspectiva\%20de\%20la\%20complejidad.pdf? sequence $=1$

Iniciativa Carta de la Tierra. (2015, 29 de octubre). La Carta de la Tierra. http://cartadelatierra.org/descubra/la-carta-de-la-tierra/

Ministerio del Medio Ambiente. (2018). Una mirada desde la institucionalidad ambiental chilena Implementación Pedagógica de la Educación Ambiental. Santiago. https://mma.gob.cl/wpcontent/uploads/2018/08/LIBRO-EDUCACION-AMBIENTALfinal_web.pdf

Montero, E. (2012). Educación a Nivel Internacional: Una propuesta para México [tesis para obtener grado de Magister. Tecnológico de Monterrey, México]. Repositorio ITESM. https://repositorio.itesm.mx/bitstream/handle/11285/629457/3306800110 4550.pdf? sequence=1\&isAllowed=y 
Muñoz-Pedreros, A. (2014). La Educación Ambiental en Chile, una tarea aún pendiente. Ambiente \& Sociedade., 17(3), pp. 177-198. Recuperado de https://www.scielo.br/scielo.php?pid=S1414$753 \times 2014000300011 \&$ script=sci_abstract\&tlng=es

Ndiaye, A., Khushik, F., Diemer, A. \& Pellaud, F. (2019). Environmental Education to Education for Sustainable Development: Challenges and Issues. International Journal of Humanities and Social Science. 9(1), 1-14. http://doc.rero.ch/record/324243

Nguyen, T. (2019). Searching for education for sustainable development in Vietnam. Environmental Education Research, 12, 1-13. http://ezproxy.ubiobio.cl:2125/ehost/detail/detail?vid=11\&sid=13e9abf2d568-46c9-adfda14ba569d3f1\%40sessionmgr102\&bdata=Jmxhbmc9ZXMmc2IOZT1laC9z $d C 1 s a X Z \mid \# A N=134560435 \& d b=a s n$

Organización de las Naciones Unidas para la Educación, la Ciencia y la Cultura. (s.f.). Educación para el Desarrollo Sostenible. https://es.unesco.org/themes/educacion-desarrollo-sostenible

Rodríguez, C., Lorenzo, O. \& Herrera, L. (2005). Teoría y práctica del análisis de datos cualitativos. Proceso general y criterios de calidad. Revista Internacional de Ciencias Sociales y Humanidades, 15, 133-154. https://www.redalyc.org/pdf/654/65415209.pdf

Serrano, E., Morales, M., \& Chaves, M. (2016). Educación Ambiental en Costa Rica: tendencias evolutivas, perspectivas y desafíos. Biocenosis, 18(1-2), 2-25. https://investiga.uned.ac.cr/revistas/index.php/biocenosis/article/view/13 82

Torres-Porras, J.\& Arrebola, J.C. (2018). Construyendo la ciudad sostenible en el Grado de Educación Primaria. Revista Eureka sobre Enseñanza y Divulgación de las Ciencias. 15(2), 2501. Recuperado de https://revistas.uca.es/index.php/eureka/article/view/3198/3871 
Sánchez, R., \& Ossa, C. (2020). Educación am biental en la escuela chilena: experiencias pedagógicas en la com una de Santa Bárbara. UCMaule, 59, julio-diciem bre, 103-128. DOI: http://doi.org/10.29035/ucm aule.59.103

Wojciech, J. (2018). Education for sustainable development-from students' and geography teachers' knowledge to educational activities. Miscellanea Geographica. 23(1). 47-52. Recuperado de http://ezproxy.ubiobio.cl:2120/ehost/pdfviewer/pdfviewer?vid=19\&sid=30 608c68-ee38-4ee6-953f-6da704c7bc82\%40sessionmgr4007 